\title{
Merging perspectives: genotype-directed molecular therapy for hereditary diffuse gastric cancer (HDGC) and E-cadherin-EGFR crosstalk
}

\author{
Dandan $\mathrm{Li}^{1}$, Winifred Lo ${ }^{1,2}$ and Udo Rudloff ${ }^{*}$
}

\begin{abstract}
Hereditary diffuse gastric cancer is a cancer predisposition syndrome associated with germline mutations of the E-cadherin gene (CDH1; NM_004360). Male CDH1 germline mutation carriers have by the age of 80 years an estimated $70 \%$ cumulative incidence of gastric cancer, females of $56 \%$ for gastric and of $42 \%$ for lobular breast cancer. Metastatic HDGC has a poor prognosis which is worse than for sporadic gastric cancer. To date, there have been no treatment options described tailored to this molecular subtype of gastric cancer. Here we review recent differential drug screening and gene expression results in c.1380del CDH1-mutant HDGC cells which identified drug classes targeting PI3K (phosphoinositide 3-kinase), MEK (mitogen-activated protein kinase), FAK (focal adhesion kinase), PKC (protein kinase C), and TOPO2 (topoisomerase II) as selectively more effective in cells with defective CDH1 function. ERK1-ERK2 (extracellular signal regulated kinase) signaling measured as top enriched network in c.1380delA CDH1mutant cells. We compared these findings to synthetic lethality and pharmacological screening results in isogenic $\mathrm{CDH}^{-/-}$MCF10A mammary epithelial cells with and without $\mathrm{CDH} 1$ expression and current knowledge of E-cadherin/catenin-EGFR cross-talk, and suggest different rationales how loss of E-cadherin function activates PI3K, mTOR, EGFR, or FAK signaling. These leads represent molecularly selected treatment options tailored to the treatment of $\mathrm{CDH} 1$-deficient familial gastric cancer.
\end{abstract}

Keywords: Hereditary diffuse gastric cancer (HDGC), Epidermal growth factor receptor (EGFR), E-Cadherin (CDH1), E-Cadherin/catenin-EGFR cross-talk, Pharmacological vulnerabilities

\section{Background}

Hereditary diffuse gastric cancer (HDGC) is a cancer predisposition syndrome which accounts for up to $19-40 \%$ of familial gastric cancers and is associated with an autosomal-dominant inheritance pattern due to germline CDH1 variants [1-3]. While initially a syndrome used to describe familial inheritance of diffuse gastric cancer, it is now recognized that the syndrome includes increased risk for lobular breast cancer (LBC), possibly colorectal cancer, and non-cancerous but significant conditions like

\footnotetext{
${ }^{*}$ Correspondence: rudloffu@mail.nih.gov

${ }^{1}$ Thoracic \& Gastrointestinal Oncology Branch, National Cancer Institute, Bethesda, MD, USA

Full list of author information is available at the end of the article
}

cleft lip palate syndrome [2-8]. Lifetime cumulative risk for gastric cancer in male $\mathrm{CDH} 1$ mutation carriers is $70 \%$ by age 80 ; similar risk for female $\mathrm{CDH} 1$ mutation carriers is $56 \%$ for diffuse gastric cancer and $42 \%$ for LBC [3]. Overall, the majority of $\mathrm{CDH} 1$ germline variants are truncating $\mathrm{CDH} 1$ mutations, followed by missense variants, and variants affecting splice sites [3, 9]. While $\mathrm{CDH} 1$ variants have been reported to affect each of the 16 exons of the gene, there is a non-random distribution with some hotspots reported including the truncating mutations c.1003C $>$ T, c.1212delC, c.1137G $>$ A, $1792 \mathrm{C}>\mathrm{T}$, or 2398delC [3, 9].

With recent advances in the understanding of the syndrome's natural history and genetics, detailed guidelines have been developed for genetic testing and preventative 
interventions via endoscopic surveillance, prophylactic gastrectomy, and breast imaging $[2,3,10]$. Despite these measures, effective systemic therapies for patients who develop HDGC malignancies remain elusive. Patients with metastatic HDGC typically receive the same, largely ineffective chemotherapies as patients with sporadic gastric cancer; however, HDGC patients experience inferior outcomes to sporadic gastric cancer or gastric cancer with non-pathogenic CDH1 mutations $[10,11]$. Thus, there remains a need for identification and selection of effective systemic agents for this unique patient subpopulation.

\section{High-throughput drug screening in cell-based models of HDGC}

As no effective systemic therapies are available for HDGC, an initial broad evaluation of potential drug targets is desirable. Our group conducted a differential high-throughput drug screen in gastric cancer cells derived from a stage IV HDGC patient with a truncating c.1380delA CDH1 germline mutation and gastric cancer cells derived from a liver lesion of a gastric cancer patient with wild type CDH1 [12]. The drug library utilized for screening was enriched for oncology compounds and contained multiple compounds per class to detect class effects. In addition, pathway enrichment was derived from differentially expressed gene set(s) (DEG) in hereditary c.1380delA HDGC cells in comparison to a panel of sporadic gastric cancer cell lines. c.1380delA CDH1mutant cells were selectively sensitive to inhibition of the EGFR effectors PI3K, mTOR, MEK, c-Src, FAK, and TOPO2 inhibition. The drug phenotype overlapped with the top two signaling networks found enriched by MetaCore analysis in c.1380delA cells [12]. The highest-ranking network predicted to be enriched in c.1380delA cells included a number of signaling regulators of the enriched epidermal growth factor receptor signaling pathway or inositol triphosphate (IP3)/diacylglyercol (DAG) signaling which directly or indirectly overlapped with the drug phenotype of enhanced sensitivity against MEK, mTOR, FAK, or PKC activity anti-PKC, c-Src kinase, and FAK activity. Table 1 lists the drug classes with selective activity in gastric cancer cells with hereditary $\mathrm{CDH} 1$ mutation compared to sporadic gastric cancer cells.

Sensitivity to PI3K, mTOR, and EGFR inhibition was independently observed in another sentinel report on this subject conducted in MCF10A cells, a non-tumorigenic mammary epithelial cell line [13]. Telford et al. performed a broad, comparative genome-wide siRNA screen of isogenic MCF10A cells with and without CDH1

Table 1 Drug sensitivities derived from in vitro models of HDGC

\begin{tabular}{|c|c|c|c|c|}
\hline & $\begin{array}{l}\text { CDH1 MCF10A (-/-) } \\
{[13]}\end{array}$ & CDH1 MCF10A (-/-) [13] & $\begin{array}{l}\text { c.1380delA CDH1 HDGC } \\
\text { [12] }\end{array}$ & c.1380delA CDH1 HDGC [12] \\
\hline & qHTS drug phenotype & $\begin{array}{l}\text { Lethality by siRNA target or } \\
\text { target ligand }\end{array}$ & qHTS drug phenotype & $\begin{array}{l}\text { Target kinase in enriched top } \\
\text { network }\end{array}$ \\
\hline \multicolumn{5}{|l|}{ Drug class } \\
\hline Pl3K inhibitor & Yes & $\begin{array}{l}\text { PIK3CA, PIK3CG, PIK3R5, } \\
\text { PIK3CB, PIK3CD, PIK3C2B }\end{array}$ & Yes & No \\
\hline AKT1 & No & AKT1 & No & No \\
\hline mTOR inhibitor & Yes & & Yes & Yes \\
\hline $\begin{array}{l}\text { EGFR and PDGFR family } \\
\text { inhibitor }\end{array}$ & Yes & PDGFD, EGFR, ERBB3, NRG1 & No & Yes \\
\hline Src kinase inhibitor & Yes & & No & Yes \\
\hline FAK inhibitor & $?$ & & Yes & Yes \\
\hline $\begin{array}{l}\text { ALK/ROS1-like tyrosine kinase } \\
\text { inhibitor }\end{array}$ & Yes & ROS1, ALK & Yes & No \\
\hline JAK family inhibitor & Yes & JAK2 & No & No \\
\hline BCL2 inhibitor & Yes & $\mathrm{BCL} 2$ & No & No \\
\hline Aurora kinase inhibitor & Yes & & Yes & No \\
\hline HDAC inhibitor & Yes & HDAC3, HDAC9, SIN3A, RERE & No & \\
\hline ROCK inhibitor & No & & Yes & No \\
\hline Protein kinase $\mathrm{C}$ inhibitor & No & & Yes & Yes \\
\hline
\end{tabular}

Quantitative high-throughput drug screening of MCF10A mammary epithelial cells vs isogenic $\mathrm{CDH} 1^{-/-}$MCF10A cells and hereditary c.1380del CDH1 gastric cancer cells vs sporadic $\mathrm{CDH} 1$ wild type SB.msgc-1 cells. Listed are vulnerabilities selective in $\mathrm{CDH} 1^{-1-}$-mutant $\mathrm{MCF} 10 \mathrm{~A}$ and c.1380del CDH1 cells, shared drug classes are highlighted in italics

Active in both c.1380delA CDH1 mutant hereditary SB.mhdgc-1 and control CDH1 wild type SB.msgc-1 gastric cancer cells 
expression. G-Protein-coupled receptor (GPCR) signaling proteins and cytoskeletal proteins were selectively lethal upon siRNA-mediated knockdown in the $\mathrm{CDH} 1^{-l-}$ null MCF10A cells. These genetic vulnerabilities overlapped with selective drug response profiles derived from a 4057 drug screen in the CDH1 isogenic MCF10A cell lines. Drugs and drug classes with increased sensitivity in the $\mathrm{CDH} 1^{-/-}$null MCF10A compared to CDH1 wild type cells included HDAC, PI3K, mTOR, JAK, BCL2, or aurora kinase inhibitors. Thus, when aligning drug phenotypes derived from both in vitro models of HDGC, $\mathrm{CDH}^{-1-}$ null MCF10A and c.1380delA CDH1 HDGC cells, gastric cancer cells with defective $\mathrm{CDH} 1$ function showed selective overlapping sensitivity to PI3K, mTOR, ALK/ROS-1 like tyrosine, and aurora kinase inhibition in both systems. Table 1 list selective genetic and pharmacological vulnerabilities in $\mathrm{CDH} 1^{-1-}$ null MCF10A and drug phenotype and enriched gene expression aberrations in c.1380delA HDGC cells. Differences in lineage and evolvement of screened cells, like pre-neoplastic primary mammary epithelial MCF10A cells versus metastatic cells derived from the ascites of a $\mathrm{CDH} 1$ germline mutation carrier with stage IV gastric cancer, or different coverages of the used drug libraries, might explain differences in observed drug phenotype like lack of HDAC inhibition, anti-Bcl2, and anti-XIAP sensitivity in the c.1380delA CDH1 cells or lack of sensitivity to MEK inhibition in the $\mathrm{CDH} 1^{-/-}$null MCF10A cells.

Indications of potential vulnerability to PI3KmTOR, or FAK inhibition are, in part, also corroborated by tissue studies on early T1a stage and > T2 lesions from CDH1-mutation carriers. Detailed pathology analysis of the early, non-proliferative intramucosal T1a lesions in prophylactic gastrectomy specimens of multiple family members with a c.1008G $>$ T CDH1 germline mutation showed as the earliest, disease-initiating change reduced or absent expression of $\beta$-actin, p120 catenin, and Lin-7 compared to surrounding mucosa with general loss of organization of adherens junctions; [14]. Loss of adhesion function in the intramucosal stage was followed upon progression towards $>$ T2 lesions by activation of c-Src kinase and FAK activation, and epithelial to mesenchymal transition (EMT). $\beta$-Catenin activation (as a result of p120 loss; measured by nuclear catenin staining) and mTOR activation (measured by staining with anti-phospho-mTOR Ser2448) was also observed in $\mathrm{T} 1 \mathrm{a}$ lesions isolated in gastrectomy specimens of $\mathrm{CDH} 1$ mutation carriers with del124_126CCCinsT, c.521dupA, and c.1565+1G >A variants [9]. These observations of activation of the c-Src-FAK axis, catenin and mTOR signaling in clinical specimens appears to be in line with the drug phenotypes derived from the in vitro models.

\section{E-Cadherin/catenin-EGFR cross talk and potential mechanisms for pharmacologic targeting}

Early broad pharmacologic screens in in vitro models of diffuse gastric cancer indicate that dysregulated EGFR receptor and downstream effector signaling may be involved in aberrant signal transduction selective for CDH1-deficient gastric cancer cells. While c-Src kinase and FAK activation might be a direct result of elevated GPCR signaling, activation of ERK signaling in addition to enhanced PI3K and mTOR sensitivity suggest activation of upstream receptor tyrosinase kinase signaling in HDGC cells. Thus, how is E-cadherin/catenin complex dysfunction able to activate EGFR signaling and how can loss of $\mathrm{CDH} 1$ function explain above signal perturbation and drug phenotype? E-Cadherin/catenin signaling has long been known a downstream effector of EGFR signaling [15-18]. Upon ligand activation, EGFR promotes loss of cellular adhesion and increased migration and invasion, among other mechanisms, through phosphorylation of E-cadherin bound $\beta$-catenin, plakoglobin $(\gamma$-catenin) and $\mathrm{p} 120 \mathrm{ctn}(\delta$-catenin 1$)$, leading to destabilization of the E-cadherin/catenin/actin complex (Fig. 1) [17, 19-21]. As suggested by observed co-localization and cooperativity of the EGFR and E-cadherin/catenin complexes in the cell membrane of epithelial cells, phosphorylated EGFR directly interacts with both $\beta$ - and $\delta$-catenins [22-25].

Recently, there is increased appreciation of reverse E-cadherin/catenin-EGFR cross-talk as part of a bidirectional signaling axis in cancer pathogenesis. Inhibition of ligand-activated EGFR signaling by E-cadherin is hereby dependent on the integrity of the extracellular domains of E-cadherin and independent of $\beta$-catenin or p120ctn binding [26]. CDH1 missense mutant cell lines derived from families with missense mutations in the extracellular domains of E-cadherin were correspondingly less able to suppress EGFR signaling than cell lines with wild type E-cadherin [27-29]. Similarly, deleting mutations (exons 8 and 9 of CDH1) affecting the extracellular cadherin-binding domains of E-cadherin show increased EGFR activation [30]. Hence, loss of suppression of EGFR signaling by lack of E-cadherin/catenin-EGFR interaction in HDGC families with CDH1 germline mutations might explain the increased sensitivity to EGFR and PI3K kinase inhibition in CDH1-deficient HDGC (Fig. 1).

The increased sensitivity to FAK inhibition, or to the c-Src kinase inhibitor saracatinib and the selective loss of viability upon GPCR knockdown in $\mathrm{CDH} 1^{-/-}$MCF10A mutant cells, might be explained by increased GPCR signaling. GPCR signaling directly activates c-Src, and increased GPCR signaling has been suggested by elevated intracellular phosphatidylinositol 4,5-bisphosphate (PIP2) and phosphatidylinositol 3,4,5-trisphosphate 


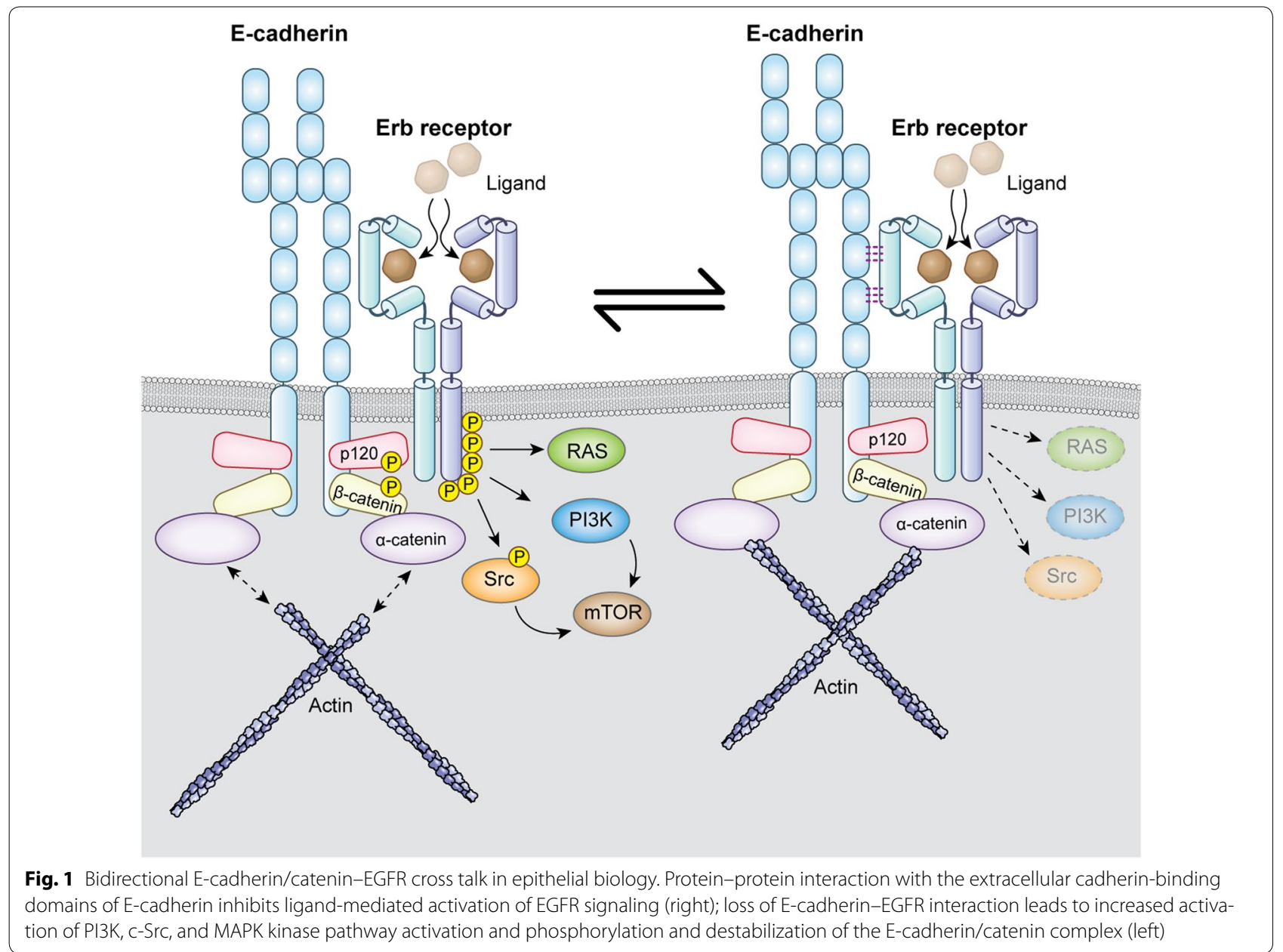

(PIP3) levels (second messenger intermediates of GPCR signaling) in c.del1380 CHH1 HDGC cells. Of note, activation of the c-Src kinase and FAK system was inferred after loss of adherens function including reduced levels of actin, p120ctn, or Lin in the progression of intramucosal T1a lesions in CDH1 germline mutation carriers [14]. Loss of p120ctn is a pro-tumorigenic driver event in epithelial cancers with augmentation of EGFR signaling in breast cancer, elevated levels and activation states of c-Src kinase and FAK have been found to be associated with accelerated progression and shorter survival in epithelial malignancies including gastric cancer [31-33]. Inhibition of the Src kinase-FAK pathway can restore cell adhesion, reduce cell migration, and promote an epithelial phenotype [34].

Activation of the EGFR downstream PI3K-mTOR pathway in CDH1-mutant cells may also be caused by the disruption of a negative feedback group of $\beta$-catenin and PTEN [35]. E-Cadherin loss is associated with enhanced nuclear $\beta$-catenin translocation, suppression of nuclear expression of EGR-1 and PTEN, and increased cytoplasmatic PI3K-AKT signaling promoting tumor cell growth (Fig. 2). A similar reciprocal relation of reduced E-cadherin expression levels and increased PI3K-AKT activation was seen in T1a lesions in prophylactic gastrectomy specimens from CDH1-mutation carriers; T1a lesions from three out of four $\mathrm{CDH} 1$ mutation carriers from different HDGC families with different truncating CDH1 mutations harbored activation of mTOR (measured by staining with anti-phospho-mTOR Ser2448) and catenin (measured by increased nuclear catenin staining) [9]. Thus, activation of PI3K-mTOR signaling in CDH1deficient gastric cancer cells might be the result of multiple signal transduction aberrations including lack of suppression of ligand-mediated EGFR activation (Fig. 1) or lack of negative feedback inhibition of the PI3K-Akt axis via reduced PTEN levels (Fig. 2).

\section{Conclusions}

Drug screening studies in isogenic $\mathrm{CDH}^{-1-}$-mutant mammary epithelial MCF10A and c.1380delA CDH1mutant gastric cancer cells show considerable overlap in 


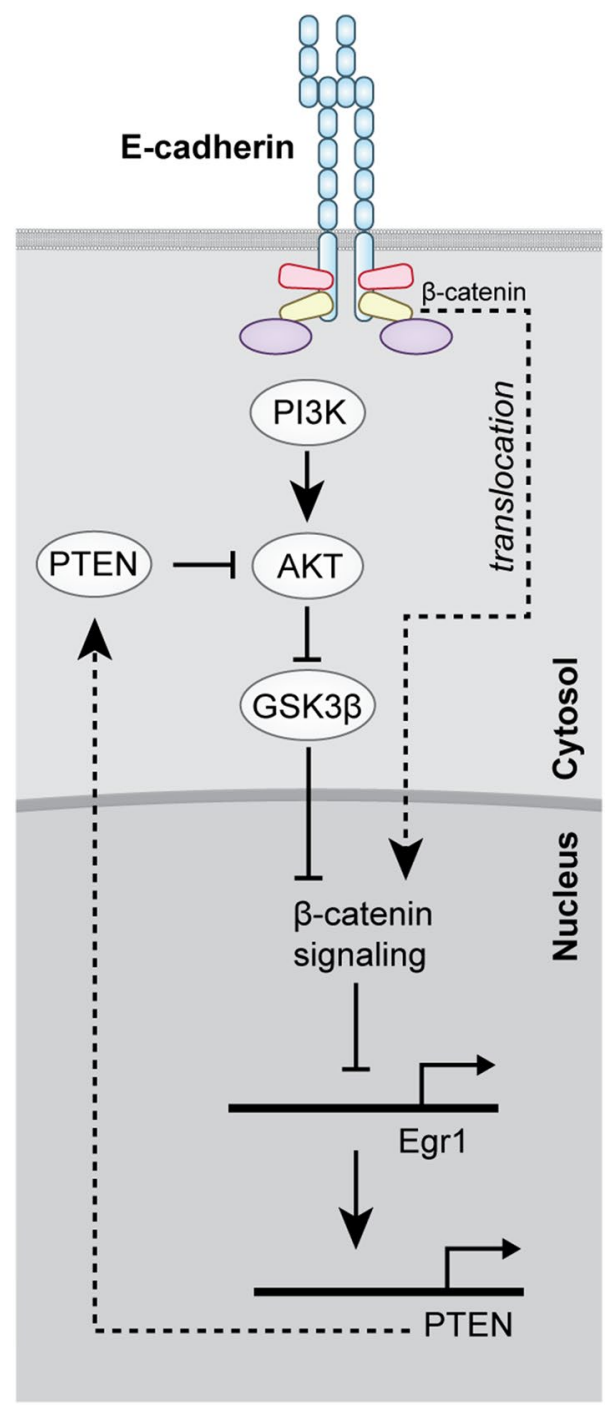

Fig. 2 E-Cadherin/catenin-PIBK/AKT crosstalk. Release of negative feedback inhibition of PI3K/AKT signaling via reduced PTEN expression through elevated nuclear $\beta$-catenin levels. Disruption of the E-cadherin/catenin complex induces nuclear translocation of $\beta$-catenin repressing Egr-1-mediated PTEN expression leading to increased AKT activation

sensitivity to PI3K, mTOR, or ALK/ROS-1 like tyrosine kinase inhibition. These pharmacological vulnerabilities are supported by comparative synthetic lethality, gene expression, and correlative tissue studies in clinical specimens of CDH1 mutation carriers, which indicate select perturbations of GPCR, actin-related, ERK1-ERK2, or FAK and c-Src kinase activity as signaling alterations and possible targets selective for CDH1-mutant gastric cancer cells. These pharmacological vulnerabilities are also supported by an improved understanding of the bidirectional cross-talk between E-cadherin/catenin and EGFR.
E-Cadherin exerts direct and indirect negative regulation onto EGFR signaling, supporting blockade of the EGFRPI3K kinase axis as therapy in this molecular subtype of gastric cancer. Considering that both anti-mTOR, antiPI3K, and anti-EGFR therapies are already in routine clinical use or in late clinical development for a number of other cancer histologies, these observations suggest that PI3K and mTOR inhibitors may be considered for molecular-targeted therapies within clinical studies for patients with HDGC in the near future.

\begin{abstract}
Abbreviations
ALK: anaplastic lymphoma receptor tyrosine kinase; $\mathrm{CDH1}$ : E-cadherin; DAG: diacylglycerol; EGFR: epidermal growth factor receptor; EMT: epithelial-mesenchymal transition; ERK: extracellular signal regulated kinase; FAK: focal adhesion kinase; HDGC: hereditary diffuse gastric cancer; IP3: inositol trisphosphate; JAK: janus kinase; MEK: mitogen-activated protein kinase; mTOR: mammalian target of rapamycin; PI3K: phosphatidylinositol 3-kinase; PKC: protein kinase C; STAT3: signal transducer and activator of transcription 3; TOPO2: topoisomerase ll.
\end{abstract}

\section{Authors' contributions}

DL conducted previous experiment with c.1380delA CDH1-mutant gastric cancer cells. DL, WL, and UR conceived and designed the clinical and translational perspectives of drug screening, compiled the figures, and wrote paper. All authors read and approved the final manuscript.

\section{Author details}

${ }^{1}$ Thoracic \& Gastrointestinal Oncology Branch, National Cancer Institute, Bethesda, MD, USA. ${ }^{2}$ Department of Surgery, University of Cincinnati College of Medicine, Cincinnati, OH, USA.

\section{Acknowledgements}

We would like to thank Ms. Erina He, MScBMC Medical Illustrator and NIH Medical Arts for compilation of the illustrations.

\section{Competing interests}

The authors declare that they have no competing interests.

\section{Consent for publication}

Not applicable.

\section{Ethics approval and consent to participate}

Not applicable.

\section{Publisher's Note}

Springer Nature remains neutral with regard to jurisdictional claims in published maps and institutional affiliations.

Received: 28 December 2017 Accepted: 31 January 2018

Published online: 22 February 2018

\section{References}

1. Kaurah P, MacMillan A, Boyd N et al (2007) Founder and recurrent CDH1 mutations in families with hereditary diffuse gastric cancer. JAMA 297:2360-2372

2. van der Post RS, Vogelaar IP, Carneiro F et al (2015) Hereditary diffuse gastric cancer: updated clinical guidelines with an emphasis on germline CDH1 mutation carriers. J Med Genet 52:361-374

3. Hansford S, Kaurah P, Li-Chang $\mathrm{H}$ et al (2015) Hereditary diffuse gastric cancer syndrome: $\mathrm{CDH} 1$ mutations and beyond. JAMA Oncol 1:23-32 
4. Pharoah PD, Guilford P, Caldas C (2001) Incidence of gastric cancer and breast cancer in $\mathrm{CDH} 1$ ( $\mathrm{E}$-cadherin) mutation carriers from hereditary diffuse gastric cancer families. Gastroenterology 121:1348-1353

5. Benusiglio PR, Malka D, Rouleau E et al (2013) CDH1 germline mutations and the hereditary diffuse gastric and lobular breast cancer syndrome: a multicentre study. J Med Genet 50:486-489

6. Richards FM, McKee SA, Rajpar MH et al (1999) Germline E-cadherin gene (CDH1) mutations predispose to familial gastric cancer and colorectal cancer. Hum Mol Genet 8:607-610

7. Frebourg T, Oliveira C, Hochain P et al (2006) Cleft lip/palate and CDH1/Ecadherin mutations in families with hereditary diffuse gastric cancer. $J$ Med Genet 43:138-142

8. Benusiglio PR, Caron O, Consolino E et al (2013) Cleft lip, cleft palate, hereditary diffuse gastric cancer and germline mutations in $\mathrm{CDH} 1$. Int J Cancer 132:2470

9. Sabesan AZB, Lo W, Wu HH, Powers A, Sorber RA, Ravichandran R, Chen I, McDuffie LA, Quadri HS, Beane JD, Calzone K, Miettinen MK, Hewitt SM, Koh C, Heller T, Wacholder S, Rudloff U (2017) Association of CDH1 germline variant location and cancer phenotype in families with hereditary diffuse gastric cancer (HDGC). J Med Genet (under review)

10. van der Post RS, Vogelaar IP, Manders P et al (2015) Accuracy of hereditary diffuse gastric cancer testing criteria and outcomes in patients with a germline mutation in CDH1. Gastroenterology 149(897-906):e19

11. Corso G, Carvalho J, Marrelli D et al (2013) Somatic mutations and deletions of the E-cadherin gene predict poor survival of patients with gastric cancer. J Clin Oncol 31:868-875

12. Chen I, Mathews-Greiner L, Li D et al (2017) Transcriptomic profiling and quantitative high-throughput (qHTS) drug screening of $\mathrm{CDH} 1$ deficient hereditary diffuse gastric cancer (HDGC) cells identify treatment leads for familial gastric cancer. J Transl Med 15:92

13. Telford BJ, Chen A, Beetham H et al (2015) Synthetic lethal screen identify vulnerabilities in GPCR signaling and cytoskeletal organization in E-cadherin-deficient cells. Mol Cancer Ther 14:1213-1223

14. Humar B, Fukuzawa R, Blair V et al (2007) Destabilized adhesion in the gastric proliferative zone and c-Src kinase activation mark the development of early diffuse gastric cancer. Cancer Res 67:2480-2489

15. Hazan RB, Norton L (1998) The epidermal growth factor receptor modulates the interaction of E-cadherin with the actin cytoskeleton. J Biol Chem 273:9078-9084

16. Ozawa M, Kemler R (1998) Altered cell adhesion activity by pervanadate due to the dissociation of alpha-catenin from the E-cadherin-catenin complex. J Biol Chem 273:6166-6170

17. Roura S, Miravet S, Piedra J et al (1999) Regulation of E-cadherin/catenin association by tyrosine phosphorylation. J Biol Chem 274:36734-36740

18. Yasmeen A, Bismar TA, Al Moustafa AE (2006) ErbB receptors and epithelial-cadherin-catenin complex in human carcinomas. Future Oncol 2:765-781

19. Lu Z, Ghosh S, Wang Z et al (2003) Downregulation of caveolin-1 function by EGF leads to the loss of E-cadherin, increased transcriptional activity of beta-catenin, and enhanced tumor cell invasion. Cancer Cell 4:499-515
20. Seton-Rogers SE, Lu Y, Hines LM et al (2004) Cooperation of the ErbB2 receptor and transforming growth factor beta in induction of migration and invasion in mammary epithelial cells. Proc Natl Acad Sci USA 101:1257-1262

21. Lilien J, Balsamo J (2005) The regulation of cadherin-mediated adhesion by tyrosine phosphorylation/dephosphorylation of beta-catenin. Curr Opin Cell Biol 17:459-465

22. Shiozaki H, Kadowaki T, Doki Y et al (1995) Effect of epidermal growth factor on cadherin-mediated adhesion in a human oesophageal cancer cell line. Br J Cancer 71:250-258

23. Jones JL, Royall JE, Walker RA (1996) E-cadherin relates to EGFR expression and lymph node metastasis in primary breast carcinoma. Br J Cancer 74:1237-1241

24. Hoschuetzky H, Aberle H, Kemler R (1994) Beta-catenin mediates the interaction of the cadherin-catenin complex with epidermal growth factor receptor. J Cell Biol 127:1375-1380

25. Bae GY, Choi SJ, Lee JS et al (2013) Loss of E-cadherin activates EGFRMEK/ERK signaling, which promotes invasion via the ZEB1/MMP2 axis in non-small cell lung cancer. Oncotarget 4:2512-2522

26. Qian X, Karpova T, Sheppard AM et al (2004) E-Cadherin-mediated adhesion inhibits ligand-dependent activation of diverse receptor tyrosine kinases. EMBO J 23:1739-1748

27. Mateus AR, Seruca R, Machado JC et al (2007) EGFR regulates RhoA-GTP dependent cell motility in E-cadherin mutant cells. Hum Mol Genet 16:1639-1647

28. Mateus AR, Simoes-Correia J, Figueiredo J et al (2009) E-Cadherin mutations and cell motility: a genotype-phenotype correlation. Exp Cell Res 315:1393-1402

29. Figueiredo J, Soderberg O, Simoes-Correia J et al (2013) The importance of $\mathrm{E}$-cadherin binding partners to evaluate the pathogenicity of E-cadherin missense mutations associated to HDGC. Eur J Hum Genet 21:301-309

30. Bremm A, Walch A, Fuchs M et al (2008) Enhanced activation of epidermal growth factor receptor caused by tumor-derived E-cadherin mutations. Cancer Res 68:707-714

31. Park JH, Lee BL, Yoon J et al (2010) Focal adhesion kinase (FAK) gene amplification and its clinical implications in gastric cancer. Hum Pathol 41:1664-1673

32. Lai IR, Chu PY, Lin HS et al (2010) Phosphorylation of focal adhesion kinase at Tyr397 in gastric carcinomas and its clinical significance. Am J Pathol 177:1629-1637

33. Schackmann RC, Klarenbeek S, Vlug EJ et al (2013) Loss of p120-catenin induces metastatic progression of breast cancer by inducing anoikis resistance and augmenting growth factor receptor signaling. Cancer Res 73:4937-4949

34. Zhang S, Yu D (2012) Targeting Src family kinases in anti-cancer therapies: turning promise into triumph. Trends Pharmacol Sci 33:122-128

35. Lau MT, Klausen C, Leung PC (2011) E-Cadherin inhibits tumor cell growth by suppressing PI3K/Akt signaling via beta-catenin-Egr1-mediated PTEN expression. Oncogene 30:2753-2766

\section{Submit your manuscript to a SpringerOpen ${ }^{\odot}$ journal and benefit from:}

- Convenient online submission

- Rigorous peer review

- Open access: articles freely available online

- High visibility within the field

- Retaining the copyright to your article

Submit your next manuscript at $\boldsymbol{\nabla}$ springeropen.com 